\title{
Cambios de volumen, área superficial y factor de forma de Heywood durante la deshidratación de cerezas (Prunus avium)
}

\author{
Mudanças de volume, área superficial e fator de forma de Heywood \\ durante a desidratação de cerejas doces (Prunus avium)
}

\author{
Antonio DE MICHELIS ${ }^{1 *}$, Beatriz Noemi PIRONE², Mabel Beatriz VULLIOUD ${ }^{2}$, \\ Mónica Roselva OCHOA², Alicia Graciela KESSELER ${ }^{2}$, Carlos Alberto MÁRQUEZ ${ }^{2}$
}

\section{Resumen}

En este trabajo, se evaluaron experimentalmente los cambios de volumen, área superficial y factores de forma durante la deshidratación de cerezas enteras. En base a los resultados obtenidos, se propusieron modelos muy simples para la evaluación matemática de dichos cambios para su uso en simulación de lechos profundos de partículas. Los cambios de volumen se pudieron representar con una ecuación lineal y los de área superficial con un polinomio de tercer orden, en ambos casos como una función de la humedad adimensional del producto. Con respecto a la evaluación de los factores de forma, se encontró que las partículas, cuanto más se deshidratan, más tienden a la forma esférica, lo cual es un hecho auspicioso, ya que no sólo el producto posee un aspecto visual agradable, sino que se habilita la utilización de la forma esférica para los efectos de adoptar una dimensión característica para la evaluación de cinéticas de deshidratación en monocapa y/o en lechos de partículas.

Palabras-clave: frutas; secado; encogimiento; factores de forma; experiencias; simulación.

\section{Resumo}

Neste trabalho foram avaliadas, experimentalmente, as mudanças de volume, área superficial e fatores de forma durante a secagem de cerejas doces inteiras. Com base nos resultados obtidos foram propostos modelos muito simples para a avaliação matemática dessas mudanças para seu uso em simulação de leitos fundos de partículas. As mudanças de volume foram representadas com uma equação linear e as mudanças de área superficial com um polinômio de terceiro grau, em ambos os casos como uma função da umidade adimensional do produto. Em relação à avaliação dos fatores de forma, foi achado que as partículas quanto mais desidratadas mais se esparramaram em forma esférica, o que é um fato favorável, já que o produto não só possui um aspecto visual agradável mas também habilita à utilização do modo esférico os efeitos de adotar uma dimensão característica para a avaliação de cinéticas de desidratação em capas magras ou em leitos fundos de partículas.

Palavras-chave: frutas; secagem e encolhimento; fatores de forma; experiências; simulação.

\section{Introducción}

Durante la deshidratación, el agua migra desde las células interiores del alimento, atravesando las membranas celulares y las paredes circundantes a las mismas, para luego fluir a través de una estructura porosa hacia el medio de secado. Los gradientes de humedad dentro del producto inducen tensiones micro estructurales, llevando el producto a encogerse y/o a deformarse. El encogimiento puede ocurrir como consecuencia de que el tejido no es capaz de retener su malla estructural cuando los espacios que contienen agua son continuamente vaciados y luego rellenados por aire, y la estructura externa colapsa. También, en el último caso, el fenómeno llamado "case hardening" (endurecimiento superficial como consecuencia de la migración de sólidos disueltos hacia la superficie de las partículas de alimento) influencia los fenómenos de encogimiento del producto (PANYAWONG; DEVAHASTIN, 2007). Evidentemente, el estudio del encogimiento en los tejidos biológicos es de una complejidad muy difícil de abordar desde el conocimiento concreto de los fenómenos físicos y químicos que ocurren durante el secado. Por ello, la gran mayoría de las contribuciones publicadas proponen estudiar fenomenológicamente el hecho real, es decir, se efectúan ensayos experimentales con condiciones de variables operativas del secado controladas y los resultados se regresionan con modelos empíricos lo más simples posible y que tienen en cuenta los cambios globales del volumen o del área superficial del producto, normalmente respecto al volumen y área superficial inicial de la muestra. Asimismo, algunos autores sostienen que no es suficiente evaluar el encogimiento de las partículas solamente en términos de los cambios adimensionales del volumen y/o del área superficial, ya que estos no tienen en cuenta los cambios de forma de las partículas, e introducen un tratamiento de los datos experimentales mediante el denominado factor de forma de Heywood

Recebido para publicação em 13/9/2006

Aceito para publicação em 19/2/2008 (001850)

${ }^{1}$ CONICET. INTA AER El Bolsón. Mármol, 1950 (8430), El Bolsón, Argentina, E-mail: ademichelis@bariloche.inta.gov.ar

2 AUVR Villa Regina, Universidad Nacional del Comahue, 25 de Mayo y Reconquista (8336), Villa Regina, Río Negro, Argentina, E-mail: cam_ing2003@yahoo.com.ar

${ }^{*}$ A quem a correspondência deve ser enviada 
(PANYAWONG; DEVAHASTIN, 2007), el cual, a través de la relación del volumen real de la partícula y el diámetro equivalente de la misma, calculado en términos del área proyectada de la partícula en su posición de reposo más estable, da idea más concreta del cambio de forma del alimento. Cuanto más se reduce el factor de forma de Heywood, más deformada aparecerá la partícula. Este tratamiento se considera necesario, ya que, por las mismas razones indicadas antes, el encogimiento no es simétrico y uniforme en la mayoría de los distintos tipos de alimentos. Este enfoque es importante, ya que, de acuerdo a la bibliografía (LOZANO; ROTSTEIN; URBICAIN, 1983; OCHOA et al., 2002a,b; PEZZUTTI, 1994; RATTI, 1994; TELIS-ROMERO; SOBRAL, 2003; MULET, 2000; MOREIRA; FIGUEIREDO; SERENO, 2000; SJÖHOLM; GEKAS, 1995), en muy buena parte de los datos publicados, el encogimiento se reporta como independiente de las variables operativas del secado, o a la sumo dependiente de la velocidad o del método de secado. En cambio, respecto de la forma, hay evidencia experimental de que la misma cambia con la velocidad y el método de secado (en cierto modo, este último está relacionado con la velocidad de secado) (PANYAWONG; DEVAHASTIN, 2007).

Por lo tanto, si bien es posible modelar cinéticas de deshidratación con modelos fenomenológicos simplificados de encogimiento global, se tendría un tratamiento más estricto si a través de la evaluación de los cambios de forma se pudiera determinar con mayor precisión la dimensión característica del alimento para el modelado. También, y aunque no se los utilice para evaluar cinéticas, los cambios de forma permitirían abordar temas importantes, como la calidad del producto final, si es posible encontrar condiciones de deshidratación que minimicen los cambios de forma.

En este trabajo, se miden los cambios de volumen y de área superficial durante la deshidratación convectiva de cerezas enteras y se proponen modelos muy simples para su utilización en simulación. Asimismo, se evalúan los factores de forma de Heywood, a los efectos de considerar si los cambios globales de volumen y área de las partículas son suficientes para estimar dimensiones características para su uso en modelado, y para evaluar como inciden los cambios de forma en la calidad visual del producto seco.

\section{Material y métodos}

\subsection{Material}

Se utilizaron cerezas frescas de la variedad Napolitana, seleccionadas y calibradas. La fruta se mantenía en cámara de frío a $4{ }^{\circ} \mathrm{C}$ y $92 \%$ de humedad relativa hasta su utilización. Las características generales de las cerezas eran:

- Sólidos solubles refractométricos: entre 16 y $21^{\circ} \mathrm{Brix}$;

- Contenido de humedad en base seca: entre 3,31 y 3,74;

- Diámetro ecuatorial: entre 0,0162 y 0,0196 m; y

- Diámetro axial: entre 0,0141 y 0,0165 m.

\subsection{Deshidratación de las frutas}

Las cerezas se deshidrataban enteras, sin pedúnculo, con flujo de aire transversal a la muestra en un equipo experimental de ciclo cerrado, con aire forzado, descrito en detalle por Ochoa et al. (2002a), que permitía regular las variables operativas. Las variables operativas utilizadas en este trabajo fueron:

- Temperatura del aire: $55 ; 60 ; 65 ; 70 ; 75$ y $80^{\circ} \mathrm{C}$;

- Velocidad del aire: 0,$1 ; 1 ; 2 ; 3$ y 5 m/s;

- Humedad relativa del aire: 5 y $50 \%$; y

- Todas las variables operativas eran medidas a la entrada de la cámara de secado.

\subsection{Medidas de las pérdidas de peso y del peso seco de las muestras}

Las pérdidas de peso de las muestras parcialmente deshidratadas se obtenían por pesada discontinua con una balanza analítica digital $( \pm 0,001 \mathrm{~g})$. El peso seco de las muestras se evaluaba secándola hasta peso constante en una estufa con aire forzado a $104^{\circ} \mathrm{C}$, con la balanza antes indicada.

\subsection{Medidas de los cambios de volumen, área superficial y factor de forma de Heywood}

Los cambios de volumen de las partículas individuales fueron determinadas mediante métodos pignométricos (desplazamiento de fluidos) y mediante medidas geométricas con un micrómetro. Los cambios de área, solamente mediante medidas geométricas y calculando el área superficial media de una esfera del mismo volumen que la partícula.

En estas medidas, se encontró que los valores obtenidos por pignometría diferían entre $-10,4$ y $9,1 \%$ respecto de los evaluados mediante medidas geométricas sobre el mismo fruto. Todos los valores informados son promedio de 12 frutos del mismo calibre en cada condición, estos promedios presentaban una desviación estándar máxima de 7,2\%.

El área proyectada de las partículas para la evaluación del factor de forma se obtenía asignando el área de un círculo equivalente del mismo diámetro mayor (el ecuatorial) que el de la fruta. La varianza máxima obtenida sobre 12 repeticiones sobre el mismo fruto era de $4,3 \%$.

\section{Resultados y discusión}

En la Figura 1 se presenta, con puntos, el cambio de volumen adimensional, experimental, $V / V_{0}(V$ es el volumen actual y $V_{0}$ el volumen inicial de la partícula), en función del contenido adimensional de humedad $X / X_{0}$ ( $X$ es la humedad actual de la muestra y $X_{0}$ su humedad inicial), y mediante líneas, las predicciones de los modelos utilizados para representarlos. Las condiciones de velocidad y humedad del aire de secado no se presentaron, ya que el análisis estadístico (ANOVA, software Origin v. 4.1) no presentaba diferencias significativas entre los distintos tratamientos. Cuando se comparaban tratamientos en condiciones extremas, por ejemplo $55^{\circ} \mathrm{C}, 50 \%$ de humedad relativa y $0,1 \mathrm{~m} / \mathrm{s}$ con $70{ }^{\circ} \mathrm{C}, 5 \%$ de humedad relativa y $5 \mathrm{~m} / \mathrm{s}$ de velocidad, se obtenía un $\mathrm{F}$ calculado (Test de Fischer, comparación de varianzas) de 0,025 y un $\mathrm{F}_{0,05}$ de 4,35 . Tampoco se encontraron diferencias significativas entre los valores obtenidos 


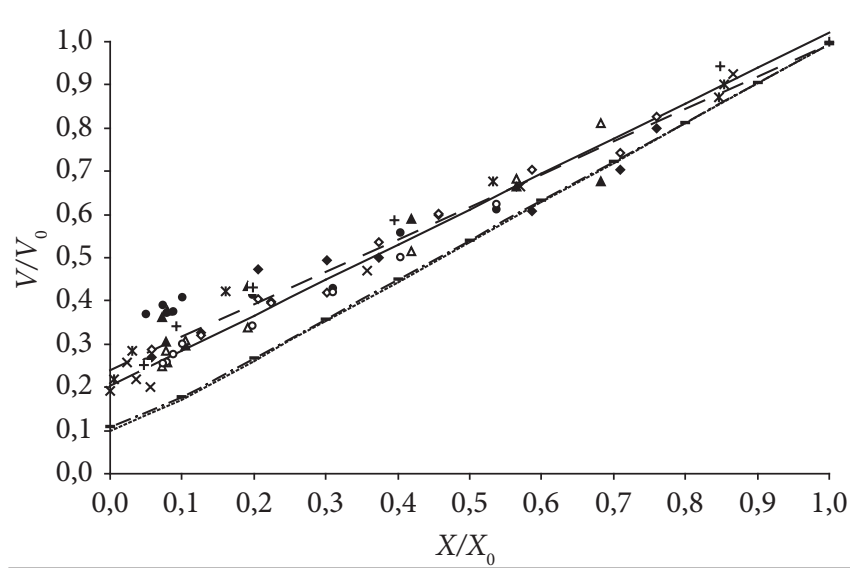

\begin{tabular}{|c|c|c|}
\hline $\begin{array}{c}\dot{\bullet} \\
+ \\
--\end{array}$ & 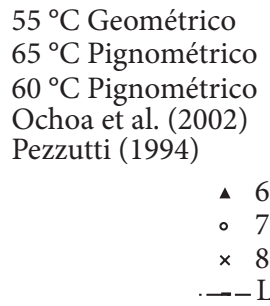 & $\begin{array}{l}\text { • } 55^{\circ} \mathrm{C} \text { Pignométrico } \\
\text { * } 75^{\circ} \mathrm{C} \text { Geométrico } \\
\text { * } 70{ }^{\circ} \mathrm{C} \text { Pignométrico } \\
\text { Este trabajo, Ecuación } 1 \\
\text { métrico } \\
\text { nométrico } \\
\text { nométrico } \\
\text { al. (1983) }\end{array}$ \\
\hline
\end{tabular}

Figura 1. Cambio de volumen adimensional de cerezas enteras durante su deshidratación en función del contenido de humedad adimensional. Valores experimentales y modelos de simulación.

por pignometría y los medidos geométricamente ( $\mathrm{F}$ calculado: 0,$\left.376 ; \mathrm{F}_{0.05}: 4,35\right) ; y$ los evaluados a las distintas temperaturas (F calculado: 0,959; $\mathrm{F}_{0,05}: 4,35$ ).

Como se observa en la Figura 1, los valores experimentales podrían ser representados por una línea recta, ya que, como se mencionó, no se encontraron diferencias significativas entre tratamientos y/o técnicas de medición. El fenómeno de encogimiento fue estudiado, entre otros, por diversos autores (LOZANO; ROTSTEIN; URBICAIN, 1983; OCHOA et al., 2002a,b; PEZZUTTI, 1994; RATTI, 1994; TELIS-ROMERO; SOBRAL, 2003; MULET, 2000; MOREIRA; FIGUEIREDO; SERENO 2000; SJÖHOLM; GEKAS, 1995), y en la mayoría de los casos, los datos experimentales fueron correlacionados con ecuaciones lineales. Pocos trabajos se han presentado en donde el encogimiento volumétrico presentaba una correlación no lineal (PANYAWONG; DEVAHASTIN, 2007). La única diferencia presentada entre los modelos lineales publicados consiste en que en la mayoría de los casos se presenta una sola línea, mientras que en algunos (LOZANO; ROTSTEIN; URBICAIN, 1983; PEZZUTTI, 1994; RATTI, 1994) se presentan dos líneas rectas con un cambio de pendiente determinado por un contenido de humedad denominado crítico, aunque estos últimos aclaran que algunos productos, al no presentar una humedad crítica, se pueden representar con una sola línea.

A los efectos de comparación, se seleccionaron los modelos publicados por Lozano, Rotstein y Urbicain (1983), Ochoa et al. (2002a) y Pezzutti (1994), que se comparan con el obtenido en este trabajo. En la Tabla 1 se presentan los descriptores estadísticos de los mencionados modelos aplicados a los datos experimentales de este trabajo.

Como se puede observar en la Tabla 1, los datos obtenidos en este trabajo se pueden representar mejor con modelos de una sola línea recta, y el modelo obtenido para el encogimiento volumétrico de cerezas enteras es (Ecuación 1):

$\frac{V}{V_{0}}=0,2407+0,7534 \frac{X}{X_{0}}$

con $\mathrm{R}^{2}=0,97$, que es comparable al modelo publicado por Ochoa et al. (2002a) pero con indicadores estadísticos levemente mejores, lo cual indica que, dentro del error experimental, los cambios de volumen de esta fruta no presentan dos rectas con distintas pendientes.

En la Figura 2 se presentan los cambios de área adimensional $a_{\mathrm{v}} a_{\mathrm{vo}}\left(a_{\mathrm{v}}\right.$ es el área actual y $a_{\mathrm{vo}}$ el área inicial de las partículas) en función de la humedad adimensional $X / X_{0}$. En este caso y de acuerdo al análisis estadístico (ANOVA, 0,05), tampoco se encuentran diferencias significativas entre tratamientos, por ello se podría indicar que los cambios de área son independientes de las variables operativas del secado.

Los cambios de área, como se ve en la Figura 2, no siguen un comportamiento lineal. Esta observación coincide con modelos previamente publicados para otros productos como papas, zanahorias y manzanas (RATTI, 1994); ajo (PEZZUTTI, 1994); frutos del Prunus cerasus (OCHOA et al., 2002a) y frutos de la Rosa eglanteria (OCHOA et al., 2002b). En algunos de los casos citados, los cambios de área dependen del tipo de alimento, de la geometría de la partícula y de la velocidad de deshidratación, pero en todos los casos los cambios de área superficial, fueron representados con un polinomio de tercer orden. Siguiendo las recomendaciones de dichos autores, los valores experimentales para cerezas enteras fueron correlacionados con el polinomio de la Ecuación 2, cuya representación (línea) se muestra también en la Figura 2:

$$
\frac{a_{\mathrm{v}}}{a_{\mathrm{vo}}}=-0,8594\left(\frac{X}{X_{0}}\right)^{3}+1,8395\left(\frac{X}{X_{0}}\right)^{2}-1,5570\left(\frac{X}{X_{0}}\right)+1,5729
$$

El coeficiente de regresión $\mathrm{R}^{2}$ fue de 0,98 y los valores experimentales diferían del modelo entre - 3,5 y 6,9\%.

En la Figura 3 se muestra el factor de forma de Heywood para cerezas parcialmente deshidratadas, y con línea llena, el valor promedio del factor de forma correspondiente al fruto fresco (obviamente, el factor de forma para el fruto fresco no depende de $X / X_{0}$, solamente es válido para $X / X_{0}=1$ ) a los efectos de comparación. El factor de forma para frutos frescos de distinto diámetro ecuatorial variaba entre 0,418 y 0,473 , diferencia porcentual de $13,3 \%$, con un promedio de 0,439 y un $\mathrm{R}^{2}$ de 0,97 . Como se puede observar, el factor de forma correspondiente a la fruta parcialmente deshidratada se mantiene prácticamente en los valores del fruto fresco para valores de humedad adimensional de 0,6 o mayores.

Mientras que para valores de contenido de humedad adimensional menores que 0,6 tiene tendencia a crecer, alcanzando valores medios de 0,52 , que son muy próximos a los valores del factor de forma correspondientes a una esfera, es decir que en 
Tabla 1. Comparación de modelos publicados con el obtenido en este trabajo para la evaluación de los cambios de volumen durante la deshidratación convectiva de cerezas.

\begin{tabular}{|c|c|c|c|c|c|}
\hline \multirow[t]{2}{*}{ Modelo de } & \multicolumn{4}{|c|}{ Error porcentual } & \multirow[t]{2}{*}{ Varianza $(\%$} \\
\hline & Máximo & Mínimo & Promedio & Promedio del valor absoluto & \\
\hline Lozano et al. (1983) & 4,10 & $-63,9$ & $-26,3$ & 27,10 & 7,2 \\
\hline Pezzutti (1994) & 3,81 & $-65,6$ & $-28,0$ & 28,2 & 7,2 \\
\hline Este trabajo, Ecuación 1 & 19,6 & $-24,9$ & $-4,0$ & 6,9 & 5,6 \\
\hline
\end{tabular}

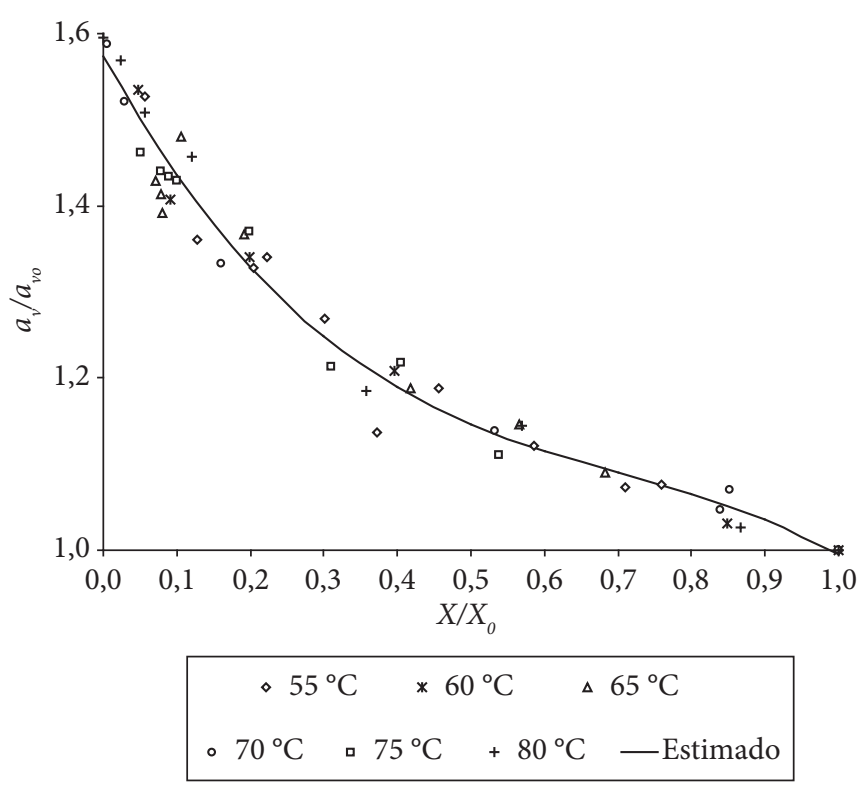

Figura 2. Cambio de área adimensional de cerezas enteras durante su deshidratación en función del contenido de humedad adimensional. Valores experimentales y modelo de simulación (línea).

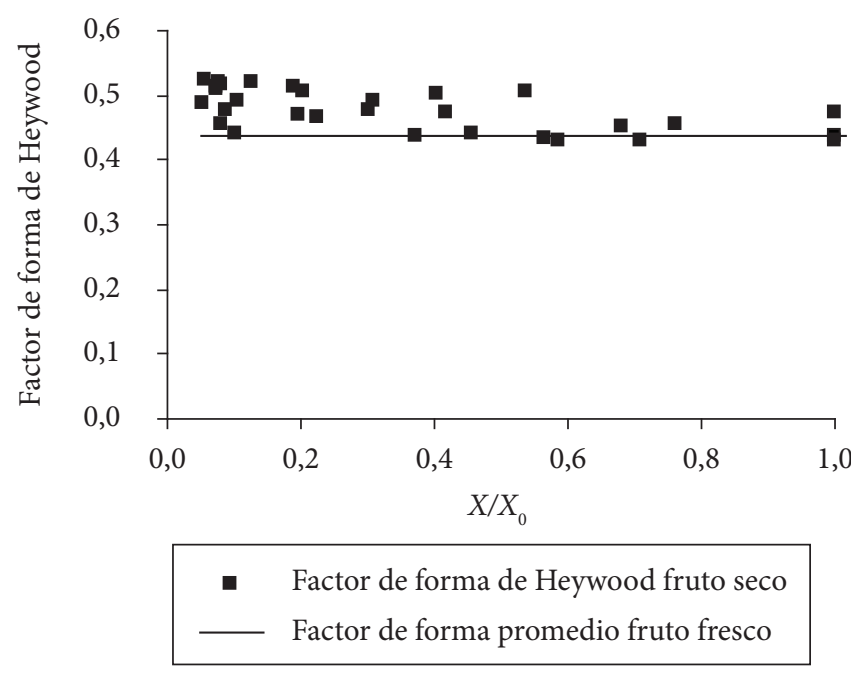

Figura 3. Cambio del factor de forma de Heywood de cerezas enteras durante su deshidratación en función del contenido de humedad adimensional. Valores experimentales. términos del factor de forma de Heywood, las cerezas deshidratadas tienden a tomar la forma esférica. El análisis estadístico (ANOVA, 0,05$)$ indica que los valores del factor de forma para $X / X_{0} 0,6$ o mayores son significativamente distintos de aquellos correspondientes a $X / X_{0}$ menores que 0,6 .

\section{Conclusiones}

Se determinaron experimentalmente los cambios de volumen, de área superficial y los factores de forma para cerezas enteras durante su deshidratación convectiva. Los cambios de área y volumen se correlacionaron con ecuaciones muy simples, siendo los cambios de volumen representados por una sola línea recta, mientras que los de área con un polinomio de tercer grado. En ambos casos, la tendencia coincide con la mayoría de los resultados publicados para alimentos. Se observaron pequeños cambios en los factores de forma de Heywood. Los cambios detectados indican que cuanto más se seca el producto, más se acerca su forma a una esfera (factor de forma de Heywood para una esfera: 0,523). Este comportamiento, además de indicar que la forma del producto final es deseable, habilita la consideración de partícula esférica para la obtención de dimensiones características necesarias para el modelado de cinéticas.

\section{Agradecimientos}

Los autores agradecen a la Universidad Nacional del Comahue - Fac. de Ingeniería - Asentamiento Universitario Villa Regina (Proyecto FAIN I125), a la ANCyPT (Proyecto PICT 0906427) y al CONICET (Proyecto PIP 5511) por la financiación de este trabajo; a CORFO CHUBUT e INTA AER El Bolsón por el apoyo logístico prestado.

El autor De Michelis es miembro del CONICET.

\section{Referencias}

PANYAWONG, S.; DEVAHASTIN, S. Determination of deformation of a food product undergoing different drying methods and conditions via evolution of a shape factor. Journal of Food Engineering, Essex, v. 78, n. 1, p. 151-161, 2007.

LOZANO, J. E.; ROTSTEIN, E.; URBICAIN, M. J. Shrinkage, porosity and bulk density of foodstuffs at changing moisture content. Journal of Food Science, New York and London, v. 48, n. 5, p. 1497-1502, 1553, 1983.

OCHOA, M. R. et al. Volume and area shrinkage during dehydration of whole sour cherry fruits (Prunus cerasus). Drying Technology, New York, v. 20, n. 1, p. 147-156, 2002 a. 
. Shrinkage during convective air drying of whole rose hip (Rosa rubiginosa L.) fruits. Lebensmittel-Wissenschaft und-Technologie, Oxford, v. 35, n. 5, p. 400-406, 2002b.

PEZZUTTI, A. Deshidratación de ajo y cebolla. Argentina, 1994. p.212. Tese - (Doutorado em Ingeniería Química). Universidad Nacional del Sur.

RATTI, C. Shrinkage during drying of foodstuffs. Journal of Food Engineering, Essex, v. 23, n. 1, p. 91-105, 1994.

TELIS-ROMERO, J.; SOBRAL, P. J. do A. Caída de presión en lecho fijo de cubos de papa, remolacha y zanahoria considerando encogimiento. Ciencia e Tecnología de Alimentos, São Paulo, v. 23, Supl., p. 110-115, dez. 2003.

MULET, A. et al. Effect of shape on potato and cauliflower shrinkage during drying. Drying Technology, New York, v. 18, n. 6, p. 1201-1219, 2000.

MOREIRA, R.; FIGUEIREDO, A.; SERENO, A. Shrinkage of apple disks during drying by warm air convection and freeze drying. Drying Technology, New York, v. 12, n. 1-2, p. 279-294, 2000.

SJÖHOLM, I.; GEKAS, V. Apple shrinkage upon drying. Journal of Food Engineering, Essex, v. 25, n. 1, p. 123-130, 1995. 